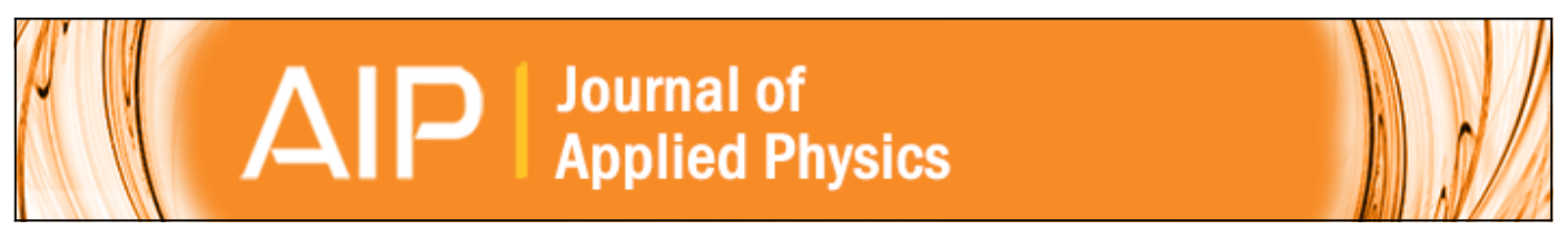

\title{
The zero-magnetization Heusler ferrimagnet
}

M. Hakimi, M. Venkatesan, K. Rode, K. Ackland, and J. M. D. Coey

Citation: Journal of Applied Physics 113, 17B101 (2013); doi: 10.1063/1.4794744

View online: http://dx.doi.org/10.1063/1.4794744

View Table of Contents: http://scitation.aip.org/content/aip/journal/jap/113/17?ver=pdfcov

Published by the AIP Publishing

\section{Articles you may be interested in}

Ferromagnetic structures in Mn2CoGa and Mn2CoAl doped by $\mathrm{Co}, \mathrm{Cu}, \mathrm{V}$, and $\mathrm{Ti}$

J. Appl. Phys. 113, 123901 (2013); 10.1063/1.4796097

Structure, magnetism, and magnetic compensation behavior of Co50-xMn25Ga25+ x and Co50- x Mn25+ x Ga25 Heusler alloys

J. Appl. Phys. 113, 103903 (2013); 10.1063/1.4794811

Design of half-metallic Heusler-based superlattices with vanishing net magnetization

J. Appl. Phys. 113, 043912 (2013); 10.1063/1.4789361

High T C half-metallic fully-compensated ferrimagnetic Heusler compounds

Appl. Phys. Lett. 99, 052509 (2011); 10.1063/1.3619844

Ab initio study on stability of half-metallic Co-based full-Heusler alloys

J. Appl. Phys. 99, 08J112 (2006); 10.1063/1.2176907

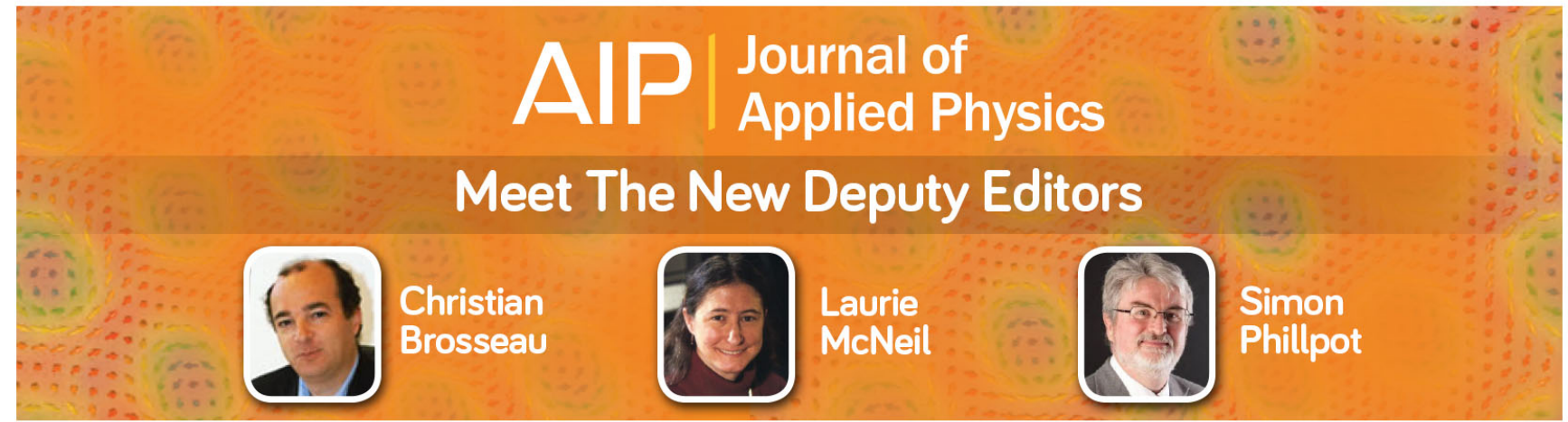




\title{
The zero-magnetization Heusler ferrimagnet
}

\author{
M. Hakimi, ${ }^{1,2}$ M. Venkatesan, ${ }^{1}$ K. Rode, ${ }^{1}$ K. Ackland, ${ }^{1}$ and J. M. D. Coey ${ }^{1, a)}$ \\ ${ }^{1}$ School of Physics and CRANN, Trinity College, Dublin 2, Ireland \\ ${ }^{2}$ Department of Physics, Isfahan University of Technology, Isfahan 84156-83111, Iran
}

(Presented 17 January 2013; received 5 November 2012; accepted 28 November 2012; published online 12 March 2013)

\begin{abstract}
An unusual zero-moment half-metallic state has been predicted in stoichiometric Heusler compounds with 24 valence electrons, where, unlike an antiferromagnet, the two sublattices are chemically or crystallographically inequivalent. We have examined the following candidate materials: $\mathrm{Cr}_{2} \mathrm{FeGe}, \mathrm{Cr}_{2} \mathrm{CoGa}, \mathrm{Fe}_{2} \mathrm{VGa}$, and $\mathrm{Mn}_{3} \mathrm{Ga}$ with various annealing and milling treatments. The $\mathrm{Fe}_{2} \mathrm{VGa}$ compound is identified as a weak itinerant ferromagnet, but both cubic $\mathrm{Mn}_{3} \mathrm{Ga}$ and perfectly ordered $\mathrm{Cr}_{2} \mathrm{CoGa}$ are potential zero-magnetization ferrimagnets. (C) 2013 American Institute of Physics. [http://dx.doi.org/10.1063/1.4794744]
\end{abstract}

An interesting proposed addition to the family of magnetic materials is the zero-moment half-metallic ferrimagnet, also known as the half-metallic antiferromagnet, or zeromoment half metal. ${ }^{1}$ This is a metallic material with a spin gap in one of the two spin subbands, so that there are only $\uparrow$ or $\downarrow$ electrons at the Fermi level. Such a half-metal could be magnetically ordered with zero net moment. It differs from a conventional ferrimagnet, such as $\mathrm{GdCo}_{3}$ at its compensation point, ${ }^{2}$ in that the magnetization vanishes over a wide range of temperature, not at just one temperature where the magnetization of the Gd and Co sublattices cancell due to their different temperature dependences.

Generally, a stoichiometric half-metal will have a spin moment which is an integral number of Bohr magnetons. All the bands are either full or empty for the spin state showing a gap in the density of states, for example, $\uparrow$. Since the total electron count in the unit cell is an integer, it follows that the number of $\downarrow$ electrons is also an integer, and so is the difference of $\uparrow$ and $\downarrow$. Hence, the spin moment of the unit cell is an integer, which may be $0,1,2 \ldots$ Bohr magnetons. The argument is valid at zero temperature and neglects spin-orbit coupling, which mixes $\uparrow$ and $\downarrow$ states and introduces an orbital contribution to the moment.

The case of interest here is $0 \mu_{\mathrm{B}}$, where the two magnetic sublattices have different chemical compositions or reside on different crystallographic sites. The Heusler compounds are a large family of ordered cubic alloys with general formula $\mathrm{X}_{2} \mathrm{YZ}^{1,3}$ where the atoms occupy four interpenetrating fcc sublattices. There are three sites in the unit cell (Figure 1(a)), $4 a, 4 b$, and $8 c$. A wide variety of occupancy schemes is possible, including $\mathrm{Y}, \mathrm{Z}, 2 \mathrm{X}\left(\mathrm{Cu}_{2} \mathrm{MnAl}\right.$-type $) ; \mathrm{Y}, \mathrm{X}, \mathrm{XZ}$ (CuHg 2 Ti-type); and random (2X, Y, Z)/4, $(2 \mathrm{X}, \mathrm{Y}, \mathrm{Z}) / 4$, $(2 \mathrm{X}, \mathrm{Y}, \mathrm{Z}) / 2$ (W-type). These are known as the $\mathrm{L} 2{ }_{1}, \mathrm{XA}$, and bcc structures, respectively. The electronic structure and magnetic properties are sensitive to the site distribution of the atoms. Even a small concentration of antisite defects in the $\mathrm{L} 22_{1}$ structure, for example, can modify the magnetic moment and Fermi-level spin polarization of the phase. ${ }^{4-6}$

\footnotetext{
a) Author to whom correspondence should be addressed. Electronic mail: jcoey@tcd.ie.
}

A Slater-Pauling plot for a series of $\mathrm{L} 2{ }_{1}$ Heusler compounds shows that the moment per unit cell is linear in electron count, with slope $-1 .^{1}$ The moment disappears when there are 24 electrons per formula, or an average of six per atom.

Two tetragonal variants are the $\mathrm{DO}_{22}\left(\mathrm{Ni}_{2} \mathrm{MnSn}\right.$ or $\mathrm{Al}_{3}$ Ti-type) structure, which has a severely distorted version of the $\mathrm{L} 2_{1}$ cubic cell, with $\mathrm{c} / \mathrm{a} \approx 1.25$, and $\mathrm{L} 1_{0}$, (AuCu-type) which is a further-deformed variant with $\mathrm{c} / \mathrm{a} \approx \sqrt{2}$ having $\mathrm{X}$ and $\mathrm{YZ}$ on alternate planes. In both unit cells, the $a$ parameter is $a\left(\mathrm{~L} 2_{1}\right) / \sqrt{ } 2$. The disorder in the $\mathrm{L} 1_{0}$ structure means that $c=c\left(\mathrm{~L} 2_{1}\right) / 2$. The cell is close to cubic with $c / a \approx 1.01$.

Here, we have made several Heusler compounds with 24 electrons. We discuss their magnetic and electronic properties, in relation to the ideal zero-moment half metal.

All alloys were made by arc-melting the constituents several times. The $\mathrm{Mn}_{3} \mathrm{Ga}$ ingots were annealed in sealed quartz tubes two weeks at temperatures in the range of $400-450^{\circ} \mathrm{C}$, and the other three compounds were annealed for 5 days at $800^{\circ} \mathrm{C}$. The ingots were then crushed or ball milled in a high-energy mill. All samples were characterized by X-ray diffraction and magnetization measurements in a $5 \mathrm{~T}$ SQUID magnetometer. The iron-containing compounds were further characterized by ${ }^{57} \mathrm{Fe}$ Mossbauer spectroscopy.

We have synthesized the 24-electron compounds $\mathrm{Cr}_{2} \mathrm{FeGe}$, $\mathrm{Cr}_{2} \mathrm{CoGa}, \mathrm{Mn}_{3} \mathrm{Ga}$, and $\mathrm{Fe}_{2} \mathrm{VGa}$. The annealed samples of $\mathrm{Cr}_{2} \mathrm{CoGa}$ and $\mathrm{Fe}_{2} \mathrm{VGa}$ exhibited a cubic Heusler structure, in accord with the previous reports, ${ }^{7,8}$ but it is difficult to decide the atomic distribution from the $\mathrm{x}$-ray powder diffraction pattern. ${ }^{1}$ However, $\mathrm{Cr}_{2} \mathrm{FeGe}$ was found to be tetragonal, although electronic structure calculations for the XA structure find it to be a zero moment half metal. ${ }^{9} \mathrm{Mn}_{3} \mathrm{Ga}$ had the tetragonal $\mathrm{D}_{22}$ structure. ${ }^{10}$ After ball milling, the $\mathrm{Mn}_{3} \mathrm{Ga}$ became almost cubic (tetragonal, with a c/a ratio 1.016 and a crystallite size of about $18 \mathrm{~nm}$. Lattice parameters for all the samples are included in Table I.

The magnetization curves for the alloys are shown in Fig. 2. The curves for unmilled $\mathrm{Mn}_{3} \mathrm{Ga}$ are sensitive to annealing treatment and show large anisotropy and hysteresis, with a saturation magnetization (inferred by doubling the extrapolated remanence) of up to $1.2 \mu_{\mathrm{B}}$ per formula unit. A little Mn 


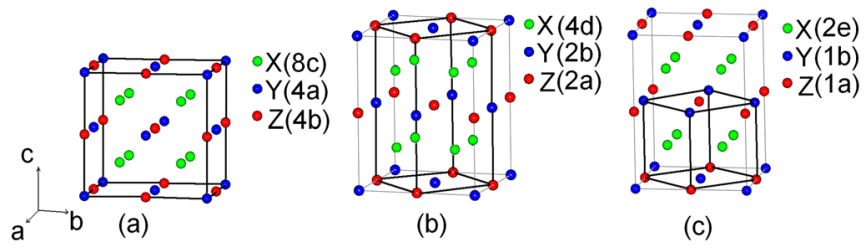

FIG. 1. Unit cells of the (a) cubic $\mathrm{L} 2_{1}$, (b), (c) tetragonal $\mathrm{D} 0_{22}$ and $\mathrm{L} 1_{0}$ structures, showing the unit cell of each compound in a black outline. In the L10 structure, $\mathrm{Y}$ and $\mathrm{Z}$ are disordered on one site. The density of the three structures is conserved in the figure.

is exsolved on annealing. The Curie temperature for this tetragonal ferrimagnet is in excess of $500^{\circ} \mathrm{C}^{1}$ The moments for all the others are much smaller, $0.07 \mu_{\mathrm{B}}$ for $\mathrm{Fe}_{2} \mathrm{VGa}, 0.25 \mu_{\mathrm{B}}$ for $\mathrm{Cr}_{2} \mathrm{CoGa}$, and $0.71 \mu_{\mathrm{B}}$ for $\mathrm{Cr}_{2} \mathrm{FeGe}$. That of $\mathrm{Fe}_{2} \mathrm{VGa}$ shows little temperature dependence but those of $\mathrm{Cr}_{2} \mathrm{CoGa}$ and $\mathrm{Cr}_{2} \mathrm{FeGe}$ are roughly doubled at $4 \mathrm{~K}$.

Mössbauer spectra of the iron-containing alloys are shown in Fig. 3. The $\mathrm{Fe}_{2} \mathrm{VGa}$ shows a single broadened absorption line in agreement with previous work. ${ }^{11}$ It is best fitted with a very small hyperfine field of $0.76 \mathrm{~T}$ at room temperature. Previous studies of this compound have attributed its weak ferromagnetic moment to antisite defects in the $\mathrm{L} 22_{1}$ structure. It is puzzling that such a small number of antisite moments (which are not detected in the spectrum) could lead to high-temperature ferromagnetism. The compound, which has a semimetallic electronic structure, with a pseudogap at the Fermi level, ${ }^{7,12-14}$ is possibly a weak itinerant ferromagnet.

The $\mathrm{Cr}_{2} \mathrm{FeGe}$ exhibits broadened magnetic hyperfine splitting, for only $25 \%$ of the iron at $20 \mathrm{~K}$ is $22.5 \mathrm{~T}$. The average hyperfine field corresponds to an iron moment of $1.5 \mu_{\mathrm{B}}$. The room temperature value is $14.5 \mathrm{~T}$, corresponding to an iron moment of $1.0 \mu_{\mathrm{B}}$ and the compound is then close to its magnetic ordering temperature. The remaining $75 \%$ of the iron is in a non cubic site with a quadrupole splitting of $0.55 \mathrm{~mm} / \mathrm{s}$. Since the measured room-temperature moment of $0.71 \mu_{\mathrm{B}} / \mathrm{f} . \mathrm{u}$ is almost three times greater than the iron contribution $\left(0.25 \mu_{\mathrm{B}}\right)$, we deduce that there is an average $\mathrm{Cr}$ moment of $0.23 \mu_{\mathrm{B}} /$ atom.

Electron count alone is in sufficient to guarantee that the zero-moment composition will be a half-metal. In order to achieve the requisite ferrimagnetic order it is necessary that there is antiferromagnetic coupling between the sublattice moments. Without this coupling, the zero-moment state will be achieved by hybridization and band broadening, which
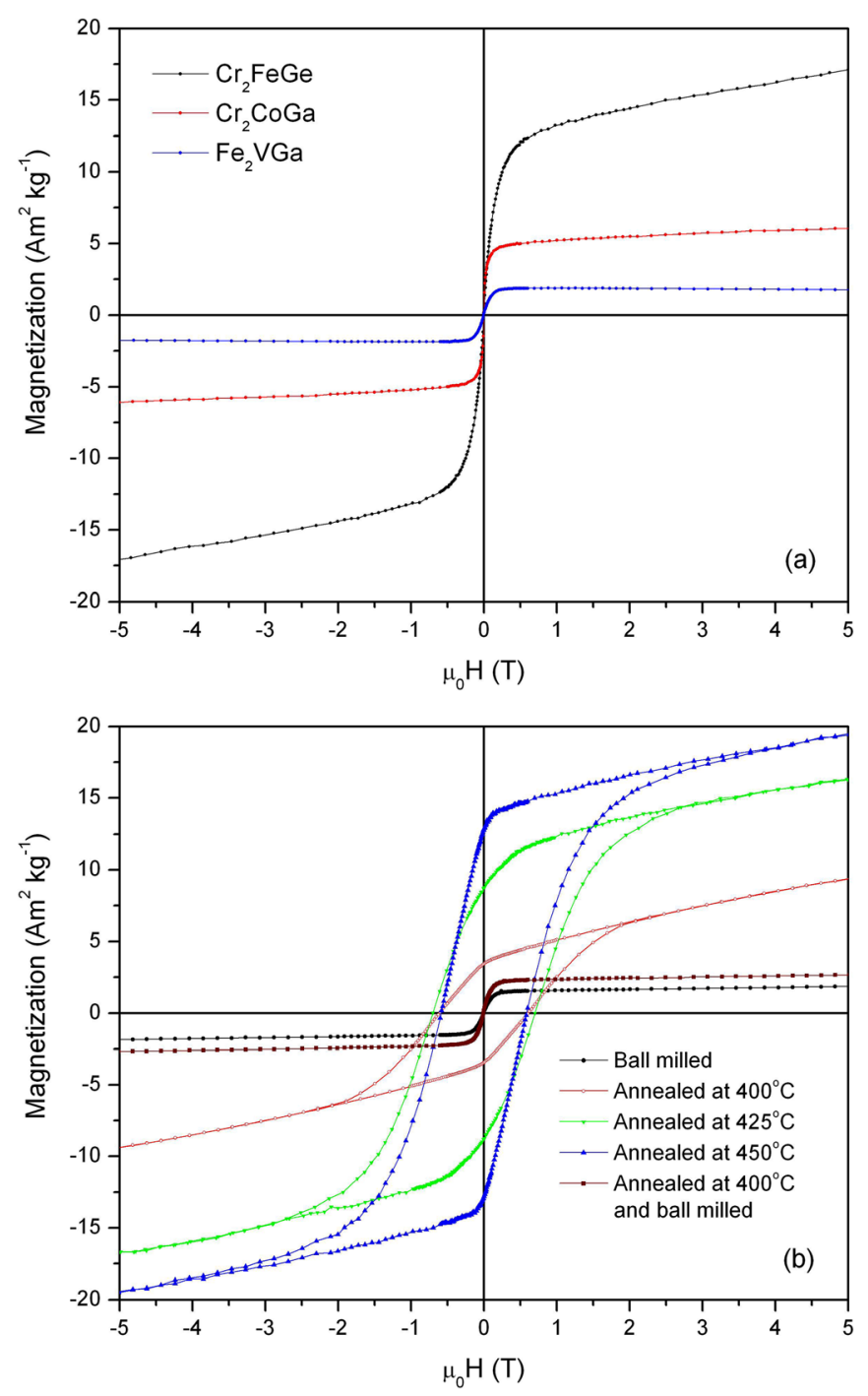

FIG. 2. Room-temperature magnetization curves for (a) three 24-electron compounds. (b) $\mathrm{Mn}_{3} \mathrm{Ga}$ after different annealing and milling treatments.

reduce the atomic moments themselves to zero, rather than their resultant. There can be a gap in the density of states of both spins, resulting in a nonmetal, rather than a half-metal. $\mathrm{Fe}_{2} \mathrm{VGa}$ is a case in point, although we raised the possibility that the tiny ferromagnetic moment of $0.035 \mu_{\mathrm{B}}$ per iron might be distributed over all the iron, and not concentrated on a few antisite defects. Using the conversion factor of $15 \mathrm{~T} / \mu_{\mathrm{B}}$ for metallic iron, the $0.76 \mathrm{~T}$ hyperfine field corresponds to an atomic moment of $0.05 \mu_{\mathrm{B}}$.

TABLE I. Magnetization and lattice parameters of the samples investigated.

\begin{tabular}{|c|c|c|c|c|c|c|}
\hline Sample & Treatment & $\mathrm{T}_{\mathrm{a}}\left({ }^{\circ} \mathrm{C}\right)$ & $\mathrm{M}_{\mathrm{s}}\left(\mathrm{Am}^{2} \mathrm{~kg}^{-1}\right)$ & $\mathrm{M}_{\mathrm{s}}\left(\mu_{\mathrm{B}} / \mathrm{f} \cdot \mathrm{u}\right)$ & $\mathrm{a}(\mathrm{pm})$ & $\mathrm{c}(\mathrm{pm})$ \\
\hline $\mathrm{Cr}_{2} \mathrm{FeGe}$ & Annealed & 800 & 17.0 & 0.71 & 462.9 & 1241.4 \\
\hline $\mathrm{Cr}_{2} \mathrm{CoGa}$ & Annealed & 800 & 6.0 & 0.25 & 579.7 & $\ldots$ \\
\hline \multirow[t]{3}{*}{$\mathrm{Fe}_{2} \mathrm{VGa}$} & Annealed & 800 & 1.7 & 0.07 & 578.0 & $\ldots$ \\
\hline & Ball milled & $\ldots$ & 1.8 & 0.07 & 377.0 & 383.6 \\
\hline & Annealed & 400 & 9.5 & 0.40 & 390.6 & 713.1 \\
\hline \multirow[t]{3}{*}{$\mathrm{Mn}_{3} \mathrm{Ga}$} & Annealed & 425 & 25.0 & 1.05 & 390.3 & 711.0 \\
\hline & Annealed & 450 & 28.5 & 1.20 & 390.2 & 710.7 \\
\hline & Annealed + ball milled & 400 & 2.7 & 0.11 & 375.8 & 382.2 \\
\hline
\end{tabular}




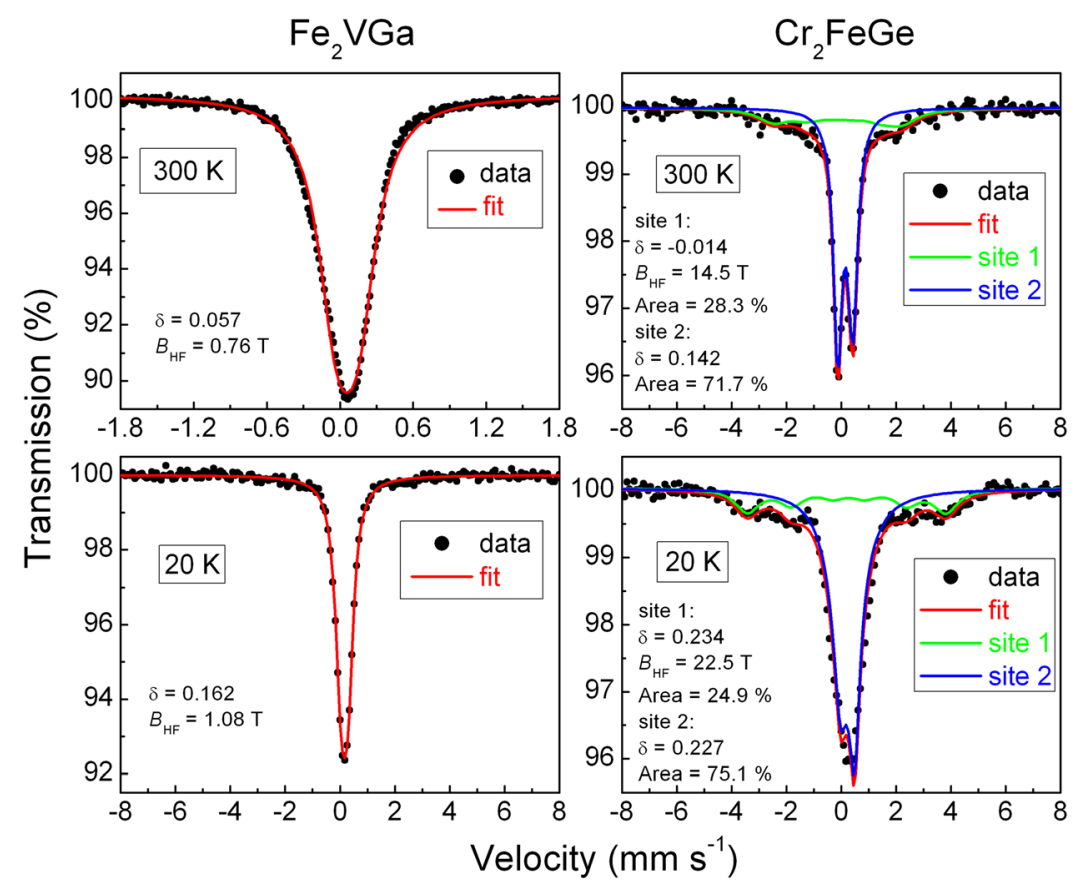

FIG. 3. Mössbauer spectra of $\mathrm{Fe}_{2} \mathrm{VGa}$ and $\mathrm{Cr}_{2} \mathrm{FeGe}$ at room temperature and $20 \mathrm{~K}$.
TABLE II. Interatomic distances in the $\mathrm{D}_{22}$ structure, in pm. The lattice parameters are $a=390 \mathrm{pm}, c=710 \mathrm{pm}$.

\begin{tabular}{cccc}
\hline \hline & $2 \mathrm{a}$ & $2 \mathrm{~b}$ & $4 \mathrm{~d}$ \\
\hline $2 \mathrm{a}$ & $390 / 450$ & $276 / 355$ & 264 \\
$2 \mathrm{~b}$ & $276 / 355$ & $390 / 450$ & 264 \\
$4 \mathrm{~d}$ & 264 & 264 & $276 / 355$ \\
\hline \hline
\end{tabular}

Antiferromagnetic direct exchange in metals is most common for atoms with seven valence electrons and a halffull $d$ shell, $4 s^{2} 3 d^{5}$. In other words, for $\mathrm{Mn}$, but $\mathrm{Cr}$ and $\mathrm{Fe}$ may also exhibit antiferromagnetic interactions in metals. The interatomic distance is quite critical. If it is too short, the $d$ band becomes too broad to support a moment. If it is too long, the direct exchange is weakened and indirect, ferromagnetic coupling becomes dominant. In the case of $\mathrm{Mn}$, the $\mathrm{Mn}$ atoms with the shortest bonds $(<250 \mathrm{pm})$ tend to be nonmagnetic, Mn with bond lengths from 250 to $280 \mathrm{pm}$ has a small moment, which tends to couple antiferromagnetically, while $\mathrm{Mn}$ with a bond length $>290 \mathrm{pm}$ tends to have a large moment, which couples ferromagnetically. This is only a rough rule of thumb, and ignores what ever else may be in the compound. Nevertheless, it is interesting to look at the interatomic distances in the cubic L2 ${ }_{1}$ structure where the $4 a$ site is occupied by nonmagnetic Ga. For $a_{0}=600 \mathrm{pm} 4 b-8 c$ $(260 \mathrm{pm})$ coupling is antiferromagnetic, but the $4 b-4 b$ $(424 \mathrm{pm})$ and $8 c-8 c(300 \mathrm{pm})$ interactions are weakly ferromagnetic.

A heavily distorted variant of the cubic structure is the tetragonal $\mathrm{D}_{22}$ structure of $\mathrm{Mn}_{3} \mathrm{Ga}$, which has $\mathrm{c} / \mathrm{a} \approx 1.29$. Table II shows how the distances change, while conserving the cell volume. ${ }^{4}$ The $2 b-4 d$ antiferromagnetic interaction is still the dominant coupling, while $4 d-4 d$ may now be close to zero. Note the $\mathrm{D}_{22}$ cell has $\mathrm{Z}=2$, whereas the $\mathrm{L} 2_{1}$ cell has $\mathrm{Z}=4$. In the $\mathrm{L} 1_{0}$ structure with the same density having unit cell parameters very close to those of milled $\mathrm{Mn}_{3} \mathrm{Ga}$, $a=376$ and $c=382 \mathrm{pm}$, the interatomic distances are $266 \mathrm{pm}$ and $268 \mathrm{pm}$; both are antiferromagnetic, so the magnetic structure will depend sensitively on the site occupancy of the nonmagnetic Ga.

We have shown that several of the 24-electron Heusler compounds exhibit a very small net magnetic moment when they have a cubic or near-cubic structure. However, the example of $\mathrm{Fe}_{2} \mathrm{VGa}$ illustrates that this does not necessarily imply they are close to zero-moment ferrimagnetism. We suggest that $\mathrm{Fe}_{2} \mathrm{VGa}$ is actually an itinerant electron ferromagnet. Alloys with $\mathrm{Mn}$ or $\mathrm{Cr}$ are most likely to exhibit antiferromagnetic interactions, and we find that a metastable, near-cubic variant of $\mathrm{Mn}_{3} \mathrm{Ga}$ has a very net small moment, but the atomic moment of the Mn is likely to be of order $2 \mu_{\mathrm{B}}$, as in the $\mathrm{D}_{22}$ structure. The other promising candidate is cubic $\mathrm{Cr}_{2} \mathrm{CoGa}$, with improved atomic order. In both cases, it is necessary to confirm by XMCD or neutron diffraction that the $\mathrm{Mn}$ or $\mathrm{Co} / \mathrm{Cr}$ moments are much greater than a few tenths of a Bohr magneton in order to be certain they are the zero-magnetization ferrimagnets we seek.

This work was supported by Science Foundation Ireland as part of the NISE project, contract No. 10/IN1.13006. Partial support from the FP7 ROMEO project is also acknowledged.

\footnotetext{
${ }^{1}$ T. Graf, C. Felser, and S. S. P. Parkin, Prog. Solid State Chem. 39, 1 (2011).

${ }^{2}$ E. Burzo, Phys. Rev. B 6, 2882 (1972).

${ }^{3}$ M. Gilleßen and R. Dronskowski, J. Comput. Chem. 30, 1290 (2009).

${ }^{4}$ D. J. Singh and I. I. Mazin, Phys. Rev. B 57, 14352 (1998).

${ }^{5}$ C. S. Lue, J. H. Ross, Jr., et al., J. Phys.:Condens. Matter 13, 1585 (2001).

${ }^{6}$ M. P. Raphael et al., Phys. Rev. B 66, 104429 (2002).

${ }^{7}$ C. S. Lue et al., J. Phys.: Condens. Matter 16, 4283 (2004).

${ }^{8}$ T. Graf et al., Z. Anorg. Allg. Chem. 635, 976 (2009).

${ }^{9}$ I. Galanakis and E. Şaşıŏlu, Appl. Phys. Lett. 99, 052509 (2011).

${ }^{10}$ H. Niida et al., J. Appl. Phys. 79, 5946 (1996).

${ }^{11}$ C. S. Lue, Y. Li, and J. H. Ross, Jr., Phys. Rev. B 67, 224425 (2003).

${ }^{12}$ R. Weht and W. E. Pickett, Phys. Rev. B 58, 6855 (1998).

${ }^{13}$ J. Goraus and A. Ślebarski, Mater. Sci. (Poland) 25, 359 (2007).

${ }^{14}$ E. Krén and G. Kádár, Solid State Commun. 8, 1653 (1970).
} 